\title{
Magneto-Optical Response Amplification In Multi-Layer Nanocomposite-Semiconductor Structures
}

\author{
Victoria Buravtsova ${ }^{1, \bullet}$,Elena Gan'shina ${ }^{1}$,Yuri Kalinin $^{2}$ and Alexander Sitnikov ${ }^{2}$ \\ ${ }^{1}$ Moscow State University, Faculty of Physics, Moscow, 119991, Russia \\ ${ }^{2}$ Voronezh State Technical University, Voronezh, 394026, Russia
}

\begin{abstract}
The spectral and field dependences of the Transversal Kerr Effect (TKE) for $\left(\mathrm{Co}_{40} \mathrm{Fe}_{40} \mathrm{~B}_{20}\right)_{Z}\left(\mathrm{SiO}_{2}\right)_{100-Z} / \mathrm{Si}$ (or $\mathrm{C}$ ) multilayer (ML) nanocomposite-semiconductor structures were studied depending on the concentration of the nanocomposite layer ferromagnetic phase $(Z)$, chemical of semiconductor layer and its thickness. It was found that under certain conditions an addition of thin nonmagnetic semiconductor layer leads to the increase of a multilayer magneto-optical (MO) response and softening of the magnetic properties of ML structure, up to the appearance of ferromagnetic (FM) ordering. The observed anomalous changes in magnetic and MO properties are well correlated with abrupt changes in the electric properties of ML structures. Decreasing of ferromagnetic phase concentration $Z$ in the nanocomposite layer as well as increasing of the semiconductor layer thickness led to the disappearance of anomalous behavior in the MO properties. The results obtained are discussed taking into account the formation of a new magnetic phase on the FM granule-semiconductor interface.
\end{abstract}

\section{Introduction}

The desire in successful materials creation for spintronics is a combination of semiconducting and magnetic properties in one material. From this perspective there is a promising way of a multilayer systems creation on the basis of ferromagnetic metal and semiconducting layers. Nevertheless, the interpretation of results from such structures is very difficult with the main complexities related to the high influence of interfaces quality and diffusion processes between dissimilar phases on the resulting properties of formed metal-semiconductor compounds [1-6]. A microstructure of the $\mathrm{FM} /$ semiconductor ML film can vary significantly, depending on the alternating layers thickness and their chemical activity; and the variations in the MO response from such ML structures also will be substantially different. As a magnetic layer a granular nanocomposite with concentrations before the percolation threshold can be used. This allows to reduce the influence of granule contacts with the neighbouring layers on macroscopic properties of the whole system and, on the other hand, to reduce the possibility of the diffusive formation of metallic-semiconductor spacers on the composite-semiconductor interface due to the presence of a barrier dielectric layer. Thus, experimental methods that can provide information about the microstructure of such materials and disclose peculiarities of the magnetic interactions inside of them become very actual.

Optical and magneto-optical methods are the most simple, effective and informative for nanostructures studies. Magneto-optical methods have several advantages, unlike optical ones, such as sensitivity to the presence of magnetic inhomogeneities, a particle shape and size changes, their volume distribution, and appearance of new magnetic phases in a nanostructure. Previous studies of nanocomposite-silicon multilayers $\left[\left(\mathrm{Co}_{45} \mathrm{Fe}_{45} \mathrm{Zr}_{10}\right)_{\mathrm{Z}}\left(\mathrm{Al}_{2} \mathrm{O}_{3}\right)_{100-\mathrm{Z}}(\mathrm{X}) / \mathrm{Si}(\mathrm{Y})\right]_{\mathrm{n}} \quad[7-9]$ with composite concentrations near and under the percolation threshold have shown that the addition of silicon layers leads to anomalous behaviour of electrical, magnetic and magneto-optical properties of ML with small thickness of Si $(<2 \mathrm{~nm})$. In [9] it was assumed that TKE spectra and anomalies in field dependences were related to competition of contributions from several magnetic phases.

In the present work we focus on MO properties of nanocomposite-semiconductor multilayers $(\mathrm{CoFeB})_{\mathrm{z}}\left(\mathrm{SiO}_{2}\right)_{100-\mathrm{z}} / \mathrm{Si}$ and $(\mathrm{CoFeB})_{\mathrm{z}}\left(\mathrm{SiO}_{2}\right)_{100-\mathrm{z}} / \mathrm{C}$ with $\mathrm{Z}=33.9$ and 40 at. $\%^{\mathrm{a}}$ in order to trace the influence of the chemical composition and the thickness of ML layers on their MO behavior.

\section{Samples and experimental details}

Nanomultilayer systems nanocomposite-silicon and nanocomposite-carbon were prepared by ion-beam deposition with different combination of granular and semiconductor layer thicknesses (see Table 1). A sketch of a model structure is shown in Fig. 1.

\footnotetext{
${ }^{a}$ The percolation threshold in the bulk nanocomposite $(\mathrm{CoFeB})_{\mathrm{z}}\left(\mathrm{SiO}_{2}\right)_{100-\mathrm{z}}$ takes place at 43 at.\%. [10].
}

- Corresponding author: v.e.buravtsova@gmail.com 


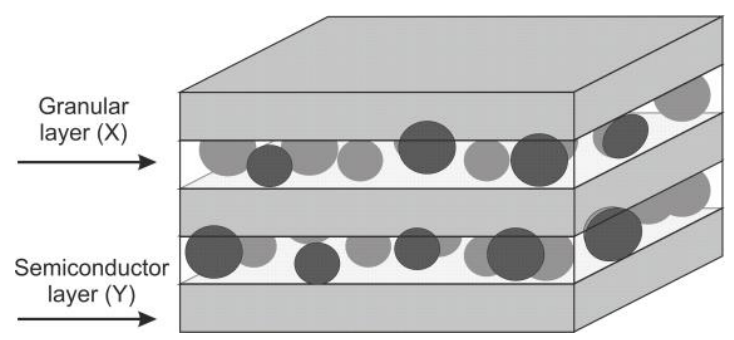

Fig. 1. Model of a nanocomposite-semiconductor multilayer structure.

By a variation of the forming layers thickness, their chemical composition and the concentration of the FM phase in the granular layers, it is possible to control the contributions from interfaces to the resulting magnetooptical response.

Magneto-optical properties of the samples have been measured in the Transversal Kerr Effect (TKE) geometry.

The value $\delta=\frac{I(H)-I(-H)}{2 I(0)}$, where $I(H)$ and $I(0)$ are the reflected p-polarized light intensities in the presence and absence of a magnetic field, respectively, was directly measured in the experiment. The TKE spectra, $\delta(h v)$, were obtained in the energy range of $h v=0.5$ $4.0 \mathrm{eV}$ at the light incidence angle of $68^{\circ}$ and in the magnetic fields up to $3.0 \mathrm{kOe}$. The TKE value depends on magnetization linearly and we have measured TKE dependences on magnetic field, $\delta(\mathrm{H})$, at some fixed energies to characterize magnetic state of the ML structures.

Table 1. Number of series, chemical composition, structure formula and thickness of based layers.

\begin{tabular}{|c|c|c|c|c|}
\hline № & Multilayer structures & $\begin{array}{c}\mathrm{X} \\
\mathrm{nm}\end{array}$ & $\begin{array}{c}\mathrm{Y}, \\
\mathrm{nm}\end{array}$ & $\begin{array}{c}\mathrm{Z}, \\
\text { at.\% }\end{array}$ \\
\hline 1 & $\left(\mathrm{Co}_{40} \mathrm{Fe}_{40} \mathrm{~B}_{20}\right)_{\mathrm{z}}\left(\mathrm{SiO}_{2}\right)_{100-\mathrm{z}}(\mathrm{X}) / \mathrm{Si}(\mathrm{Y})$ & $\begin{array}{l}2.8 \\
- \\
4.8\end{array}$ & $\begin{array}{l}0.2 \\
- \\
3.5\end{array}$ & 40 \\
\hline 2 & $\left(\mathrm{Co}_{40} \mathrm{Fe}_{40} \mathrm{~B}_{20}\right)_{\mathrm{z}}\left(\mathrm{SiO}_{2}\right)_{100-\mathrm{z}}(\mathrm{X}) / \mathrm{Si}(\mathrm{Y})$ & $\begin{array}{l}2.0 \\
- \\
4.9\end{array}$ & $\begin{array}{l}0.2 \\
- \\
4.5\end{array}$ & 33.9 \\
\hline 3 & $\left(\mathrm{Co}_{40} \mathrm{Fe}_{40} \mathrm{~B}_{20}\right)_{\mathrm{z}}\left(\mathrm{SiO}_{2}\right)_{100-\mathrm{z}}(\mathrm{X}) / \mathrm{C}(\mathrm{Y})$ & $\begin{array}{l}2.8 \\
- \\
7.9\end{array}$ & $\begin{array}{l}0.2 \\
- \\
1.9 \\
\end{array}$ & 33.9 \\
\hline
\end{tabular}

\section{Results}

Field, spectral and isoenergetic TKE dependences for multilayer structures from the first series (with the concentration of the magnetic phase $\mathrm{Z}$ in the granular layer near the percolation threshold) are shown in Figure 2 and 3. It was found that an addition of a thin nonmagnetic semiconductor interlayer leads to anomalous behaviour of resulting magnetic and magneto-optical properties of the multilayer structure of this series. It can be seen that with silicon thickness increasing the shape of the magnetization curve evolves from superparamagnetic $(\mathrm{Y}=0.19 \mathrm{~nm})$ to ferromagnetic one $(\mathrm{Y}=0.92,2.47 \mathrm{~nm})$ with the saturation field of less than $1 \mathrm{kOe}$ (Fig. 2A). Meanwhile, the negative effect amplitude grows (Fig. 2B) and reaches its maximum at $\mathrm{Si}$ thickness of approximately $0.6 \mathrm{~nm}$. This is also clearly seen at the isoenergetic dependences of the effect on Si thickness (Fig. 3A). A further increase of the interlayer thickness leads to a weakening of the effect and a reduction of the magnetization curve back to the superparamagnetic type. The spectrum shape changes as well: the effect becomes positive over the entire wavelength range, the spectrum shape becomes similar to the bulk nanocomposite with $\mathrm{CoFeB}$ granules in a complex $\mathrm{SiO}_{2}+\mathrm{Si}$ matrix or/and silicides like in [11].
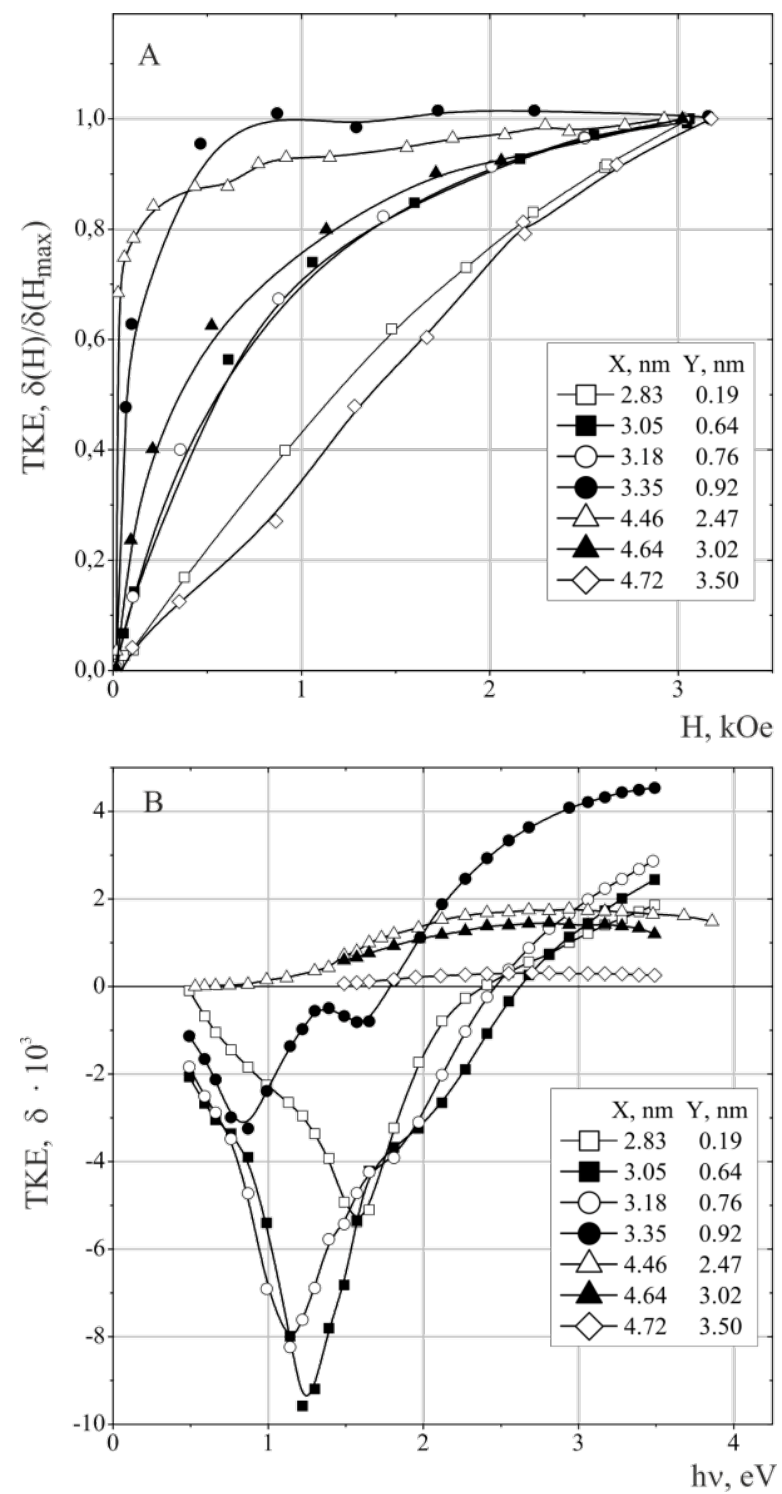

Fig. 2. Normalized TKE magnetic field dependences $\delta(\mathrm{H}) / \delta\left(\mathrm{H}_{\max }\right)$ for the wavelength $\mathrm{h} v=2 \mathrm{eV}(\mathrm{A})$, and TKE spectra (B) for series 1.

Such behavior of the first ML series can be explained by a fact that a new nanocomposite $(\mathrm{CoFeB})$ in a complex matrix $\left(\mathrm{SiO}_{2}+\mathrm{Si}\right)$ is formed on the interface of ferromagnetic granule-silicon, as in the case considered in [11]. The percolation threshold in this new nanocomposite is reached at approx. $0.6 \mathrm{~nm}$ silicon thickness. For the same silicon thickness one can see that TKE in the near-IR range reaches its maximum value 
and there is a good correlation with the beginning of the electrical percolation (Fig.3 B).

The formation of $\mathrm{Si}$ layer occurs over the $\mathrm{CoFeB}$ granules due to the surface energy difference between metal granules and the composite layer matrixes [7]. The first atomic layer of the semiconductor can cooperate with metal in the form of silicide with a high probability, and then can grow on a surface in the form of island structures. At the early stage of semiconductor island growth, when the surface area occupied by the islands is smaller, than that occupied by metallic granules emerging at the interface two channels of electrotransport are possible. The first is a granuleinsulator-granule way, as in a common bulk granular nanocomposite. The second (new) way of electrotransport is a granule- semiconductor-insulatorgranule channel. The resulting island structure of the semiconductor layer will have a minor impact on the resistivity of the whole ML up to a thickness at which the semiconductor layer becomes continuous over a distance larger than the intergranular spacing. Under such conditions, granule-spacer-granule electrotransport channels arise and the resistivity sharply drops (Fig3. model II). So the percolation threshold comes in the granule-semiconductor-granule and/or granule(silicide+semiconductor)-granule systems. Such percolation transition is responsible for the increase of the effective magnetic interaction between ferromagnetic granules through the semiconductor layer (or a silicide) what explains the observed features in the magnetic and MO properties of the samples with small thickness of $\mathrm{Si}$ layer. Obtained results allow to conclude that MO response in the studied ML are formed both by magnetic granular layer and new magnetic phase formed on the magnetic granule - semiconductor interface.

Further growth of semiconductor spacers causes a separation of the nanocomposite layers and their interaction through the semiconductor becomes weaker and amplification of the MO response disappears. The insignificant growth of the ML electrical resistivity is determined mainly by the silicon conductivity in this range of the semiconductor thickness (Fig. 3. ML model III).

In the second series of ML the magnetic phase concentration $\mathrm{Z}$ within the composite layers was slightly less than in the first one (farther away from the percolation), but it leads to abrupt changes in the MO properties. TKE value for series 2 (Fig.4) not only did not grow, but fell more than an order of magnitude compared to the nanocomposite $\left(\mathrm{Co}_{40} \mathrm{Fe}_{40} \mathrm{~B}_{20}\right)_{33,9}\left(\mathrm{SiO}_{2}\right)_{66,1}$ (witness). Maximal magnetooptical response for series 2 structures coincides with a beginning of the electrical percolation at the region near $0.5 \mathrm{~nm}$ of silicon thickness. Nevertheless, the effect is almost negligible, so it was possible to obtain TKE field dependencies only for several samples. All these dependences $\delta(\mathrm{H})$ show a paramagnetic type of magnetization for ML structure.

Such MO behavior testifies that a decrease of the ferromagnetic phase concentration in the nanocomposite layer leads to an increase of distances between the granules both inside of the nanocomposite and on the interface. In this case, the exchange interaction between granules of the adjacent layers through the semiconductor spacer weakens and it causes a decline of contribution from a new FM phase formed at the boundary of the FM granule-semiconductor in resulting MO response. It leads to intergranular exchange interaction attenuation, thus, $\mathrm{MO}$ response amplification vanishes. Moreover, the formation of non-magnetic silicides on the FM granule - silicon interface causes further TKE reduction [6].
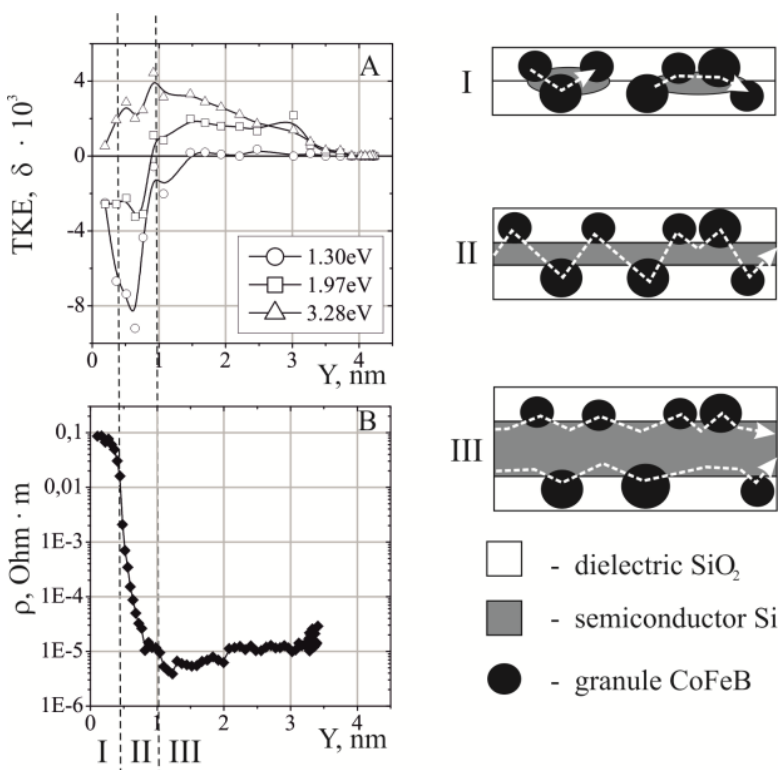

III

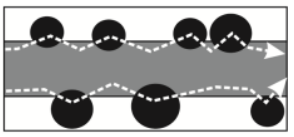

$\square$ - dielectric $\mathrm{SiO}_{2}$

$\square$ - semiconductor $\mathrm{Si}$

- granule $\mathrm{CoFeB}$

Fig. 3. Isoenergetic Si thickness dependences of TKE (A), electric resistance (B) for series 1, and ML models for three specific ranges of the thickness.

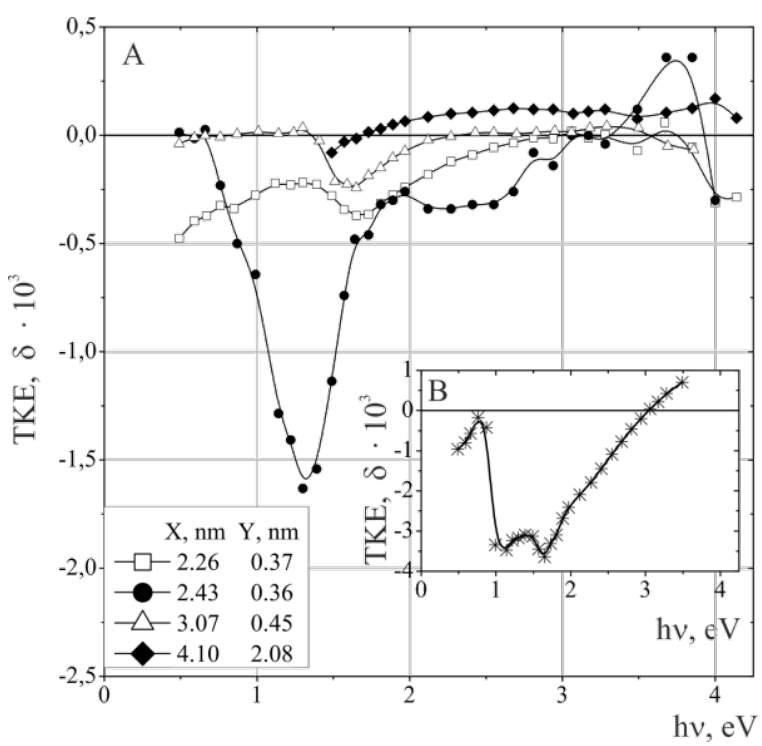

Fig. 4. TKE spectra for ML second series (A) and witness deposited layer-by-layer composite $\left(\mathrm{Co}_{40} \mathrm{Fe}_{40} \mathrm{~B}_{20}\right)_{33,9}\left(\mathrm{SiO}_{2}\right)_{66,1}$ with based layer thickness $\mathrm{X}=3.2 \mathrm{~nm}(\mathrm{~B})$.

For the third ML series, where silicon was replaced by carbon, the studies of TKE spectra revealed that the shape of $\delta(h v)$, curves were generally very similar to TKE spectra shape for witnesses (granular nanocomposite of the same chemical composition). The 
magnitude of MO signal was increasing with increasing of the granular composite layer thickness (X) and decreasing of carbon layer thickness (Y). It was found that adding of a thin non-magnetic semiconductor layer leads to the multilayer MO response decrease as compared to the nanocomposite witness (Fig. 5).
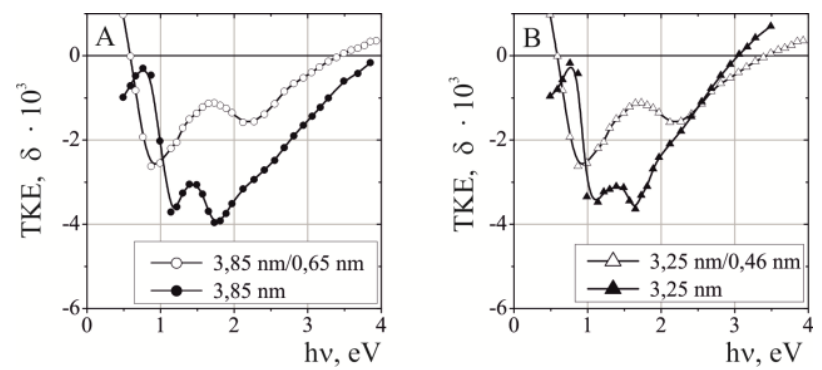

Fig. 5. TKE spectra for nanocomposite witness $\left(\mathrm{Co}_{40} \mathrm{Fe}_{40} \mathrm{~B}_{20}\right)_{33,9}\left(\mathrm{SiO}_{2}\right)_{66,1}$ without carbon interlayer (black marker) and for series $3 \mathrm{ML}$ with the same thicknesses of granular layers $(\mathrm{X})$.

TKE field dependencies exhibit a superparamagnetic kind of magnetization and practically matched. This fact demonstrates that $\left(\mathrm{Co}_{40} \mathrm{Fe}_{40} \mathrm{~B}_{20}\right)_{33,9}\left(\mathrm{SiO}_{2}\right)_{66,1}$ composite in the ML structure of the third series is superparamagnetic, and ferromagnetic granules do not interact. Electric resistance of the third ML series with carbon layers thickness ranging from 1 to $1.6 \mathrm{~nm}$ abruptly decreases. Apparently, the electrical percolation transition is observed in the ML.

With the increase of carbon spacer thickness carbon bridges begin to be formed between the ferromagnetic granules, protruding from the composite layers. These carbon bridges merge into a continuous layer at the carbon thickness about $1.7 \mathrm{~nm}$. The formation of the latter layer leads to the electrical resistivity percolation, whereas no sharp TKE magnitude changes occur at this thickness.

The ML MO response considerably weakens as $\mathrm{C}$ is being replaced with $\mathrm{Si}$. The decrease of magneto-optical response could be associated with the higher chemical activity of $\mathrm{Si}$ compared with $\mathrm{C}$ as well as with the mutual compensation between TKE of the base composite layer and TKE of a new composite formed at the "FM granule-semiconductor" interface with $\mathrm{Si}$, which may have opposite signs [11]. The assumption of mutual compensations is confirmed by positive sign of TKE signal in whole investigated wavelength range from samples with the thickest silicon layers (Fig.4).

\section{Conclusion}

The spectral and field dependences of the Transversal Kerr Effect for $\left(\mathrm{Co}_{40} \mathrm{Fe}_{40} \mathrm{~B}_{20}\right)_{\mathrm{Z}}\left(\mathrm{SiO}_{2}\right)_{100-\mathrm{Z}} / \mathrm{Si}$ (or $\mathrm{C}$ ) multilayer nanocomposite-semiconductor structures with different thicknesses of nanocomposite and semiconductor layers as well as magnetic phase concentrations $\mathrm{Z}$ have been studied. It has been found that $\mathrm{MO}$ response from studied $\mathrm{ML}$ is formed by magnetic composite layer and a new magnetic phase formed on the magnetic granule-semiconductor interface. The contribution from the new magnetic phase to the resulting MO response depends on thicknesses of nanocomposite and semiconductor layers and magnetic phase concentration $\mathrm{Z}$ within the nanocomposite layer. Increase of a semiconductor layer thickness leads to the continuous granule-semiconductor-granule and/or granule-(silicide+semiconductor)-granule chain formation and causes the appearance of transport percolation transition in nanocomposite-semiconductor structures. Such a percolation transition is induced by increasing of the effective magnetic interaction between $(\mathrm{CoFeB})$ granules through the semiconductor (or a silicide) layer and explains anomalous behavior of observed magnetic and MO properties of structures with thin Si layers and, at the same time, short distances between FM granules within the composite layer (where $\mathrm{Z}$ is near percolation). At lower FM phase concentrations $\mathrm{Z}$ as well as an increase of a layer thickness of the semiconductor, the distance between the granules become larger, so it leads to the exchange interaction weakening between granules. This explains TKE reduction and anomalous behavior of the MO properties of ML disappearance.

The contribution from the new phase at the interfaces is considerably reduced as $\mathrm{Si}$ is being replaced with $\mathrm{C}$, and can be associated with the higher chemical activity of $\mathrm{Si}$ compared with $\mathrm{C}$.

This research was supported by the Russian Foundation for Basic Researches №15-02-02077

\section{References}

1. V. O. Vas'kovskiya, G. S. Patrin, et.al. Physics of the Solid State 49, 302 (2007)

2. S.M. Valvidares, C. Quiros, et.al. Phys. Rev. Lett. B 78, 064406 (2008)

3. I. I. Pronin, M. V. Gomoyunova, et. al. Physics of the Solid State 53, 606 (2011)

4. I. V. Belousov, G. V. Kuznetsov, O. P. Pchelyakov. Semiconductors 40, 881 (2006)

5. M. V. Gomoyunova, G. S. Grebenyuk, et.al. Physics of the Solid State 55, 437 (2013)

6. S. R. Naik, S. Rai, et. al. J. Phys. D: Appl. Phys. 41, 115307 (2008)

7. E. A. Gan`shina, N. S. Perov, et. al., Bulletin of RAS: Physics. 72, 1379 (2008)

8. V. Buravtsova, E. Gan'shina, E. Lebedeva, et. al. SSP 168, 533(2011)

9. E. Gan'shina, V. Buravtsova, A. Novikov, et .al. SSP 190, 361 (2012)

10. E.A.Gan'shina, M.V.Vashuk, A.N.Vinogradov et al. JETP 98, 1027 (2004)

11. V. Buravtsova, E. Gan'shina, Yu. Kalinin, A. Sitnikov, D. Zubakin. SSP 233, 603(2015) 